\title{
O processamento auditivo e a combinação de traços distintivos na aquisição de fala em crianças com desvios fonológicos
}

\author{
Auditory processing and combination of distinctive features in \\ speech acquisition in children with phonological disorders
}

\author{
Victor Gandra Quintass ${ }^{1}$, Tiago Mendonça Attoni ${ }^{1}$, Márcia Keske-Soares ${ }^{1}$, Carolina Lisbôa Mezzomo ${ }^{1}$
}

\begin{abstract}
RESUMO
Objetivo: Investigar a relação entre as habilidades auditivas e as combinações de traços presentes na fala de crianças com desvios fonológicos. Métodos: Participaram da pesquisa 22 crianças com diagnóstico de desvio fonológico, com idades entre 5 e 7 anos e de ambos os gêneros. Foi feita a coleta dos dados de fala por meio do teste Avaliação Fonológica da Criança. A combinação de traços foi observada mediante o Modelo Implicacional de Complexidade de Traços (MICT). Os traços adquiridos consideraram uma produção correta acima de $80 \%$. Foram aplicados ainda os testes: Avaliação Simplificada do Processamento Auditivo (triagem), Teste Dicótico de Dissílabos Alterados (SSW), Teste Dicótico de Dígitos, Teste de Fusão Binaural, Teste de Fala no Ruído e o Teste de Logoaudiometria Pediátrica (PSI). Resultados: Todas as crianças obtiveram 100\% de êxito no PSI. Nos testes de fala no ruído e de fusão binaural, bem como na triagem, os sujeitos também obtiveram médias altas. Os testes SSW e Dicótico de Dígitos mostraram escores baixos. A combinação de traços que se mostrou mais alterada foi a [+aproximante,+contínuo], que está na composição do fonema /r/, seguida de [coronal,+contínuo]/(-anterior), que compõem os fonemas /, $3 /$. Conclusão: Os resultados obtidos nos testes SSW e Dicótico de Dígitos mostraram haver alteração nas habilidades de ordenação temporal complexa e figura-fundo, bem como memória sensorial defasada. Ao relacionar com os traços, estas alterações encontradas podem comprometer a aquisição destes traços descritos.
\end{abstract}

Descritores: Distúrbios da fala; Transtornos da audição; Percepção auditiva; Transtornos da percepção auditiva; Testes auditivos; Criança

\section{INTRODUÇÃO}

Os fonemas não são segmentos indivisíveis, mas sim o resultado do conjunto de propriedades que caracterizam a sua produção. Essas unidades mínimas, descritas na fonologia autossegmental, são chamadas de traços distintivos e têm por finalidade descrever as propriedades articulatórias e/ ou acústicas que entram na composição do som, diferenciar itens lexicais e agrupar os sons em classes naturais, ou seja, grupos de fonemas que possuem correlação entre si e sofrem as mesmas mudanças ou regras fonológicas ${ }^{(1)}$.

Para alguns autores, o processo de aquisição fonológica ocorre de maneira gradual, até que haja o estabelecimento do

Trabalho realizado no Programa de Pós-Graduação em Distúrbios da Comunicação Humana, Universidade Federal de Santa Maria - UFSM - Santa Maria (RS), Brasil.

(1) Programa de Pós-Graduação em Distúrbios da Comunicação Humana, Universidade Federal de Santa Maria - UFSM - Santa Maria (RS), Brasil. Endereço para correspondência: Victor Gandra Quintas. Av. Dr. Guilhermino de Oliveira, 466, Contagem (MG), Brasil, CEP: 32.341-290. E-mail: victorquintas@ymail.com

Recebido em: 4/1/2010; Aceito em: 31/8/2010 sistema fonológico, de acordo com a comunidade linguística em que a criança está inserida. A idade esperada para o estabelecimento deste sistema fonológico é em torno dos 5 anos, podendo estender-se até, no máximo, os 6 anos de idade ${ }^{(2)}$.

Quando os traços distintivos não são adquiridos de forma combinada, poderá ocorrer o desvio fonológico que também pode ser chamado de distúrbio ou transtorno fonológico. Nesta pesquisa, será usado o termo desvio fonológico (DF), devido à maior ocorrência na literatura pesquisada ${ }^{(1)}$.

Há evidencias de que os traços são agrupados em unidades funcionais que poderiam ser chamados de "classes naturais" de traços. Estes traços permitem, além de agrupar fonemas, também diferenciar um fonema do outro ${ }^{(1,3)}$.

O Modelo Implicacional de Complexidade de Traços (MICT), construído a partir dos dados de 25 sujeitos com atraso na aquisição fonológica, na faixa etária de 4 a 10 anos, representa as relações de implicação entre os traços, formando caminhos a serem percorridos durante a aquisição fonológica ${ }^{(4)}$.

A autora refere que na aquisição a criança apresenta uma estrutura representacional básica (traços não-marcados) e à medida que a aquisição ocorre, o input e as próprias capacidades 
cognitivas e articulatórias influenciarão as especificações de outros traços (traços marcados), que não estavam presentes na representação. Estas especificações acontecem gradativamente, tanto em termos de quais traços são especificados primeiro, como em termos de expansão desses no sistema ${ }^{(4)}$.

No entanto, o aumento de complexidade não se dá da mesma forma para todas as crianças, pois elas não seguem a mesma rota de aquisição, mas percorrem diferentes caminhos para atingir o alvo adulto. A autora propõe o MICT, iniciando com o Estado 0 de complexidade (composto por traços nãomarcados). Os caminhos a serem percorridos, inicialmente, são três, seguindo-se de acordo com a especificidade da criança ${ }^{(4)}$.

Quanto mais distante do ponto zero estes estiverem, mais complexos (ou mais marcados) são os traços. Se em um mesmo caminho existem dois ou mais traços ou combinações de traços, significa que entre eles há uma relação de implicação. Nesse caso, para que um traço que está mais abaixo no caminho, seja especificado, é preciso que os traços que estão mais acima também o sejam. Se um traço ou uma combinação de traços é alvo de dois ou mais caminhos convergentes, isso quer dizer que, para que este traço seja especificado, é necessário que o conjunto de traços correspondentes aos caminhos convergentes já tenham sido especificados ${ }^{(4)}$.

Desta forma, as crianças que não partilham destes caminhos, ou que por algum motivo apresentam alterações nos traços descritos, apresentam os chamados desvios fonológicos ${ }^{(4)}$.

O desvio fonológico é definido como uma dificuldade de fala, caracterizada pelo uso inadequado de sons, de acordo com a idade e com as variações regionais, que podem envolver erros na produção, percepção ou organização dos sons. A fala desviante é caracterizada por: substituições, omissões, inserções, transposições e ou distorções dos sons da língua ${ }^{(5-7)}$.

Embora o desvio fonológico desperte grande interesse por parte de pesquisadores, suas causas são indefinidas. No entanto, acredita-se que muitos fatores, tais como gênero, idade, alterações auditivas e aspecto familial, podem influenciar o aparecimento desta desordem ${ }^{(5-10)}$.

Acredita-se que dificuldades na língua oral podem estar intimamente relacionadas às desordens do processamento auditivo (PA), pois a audição é a principal via de entrada para a aquisição da mesma ${ }^{(11,12)}$.

O PA é, portanto, o conjunto de habilidades necessárias para processar o sinal sonoro, que envolve não somente as habilidades, mas também o comportamento auditivo periférico. Assim, uma perda auditiva pode levar o indivíduo a ter déficit do PA, mas uma dificuldade no PA não é indicio de aparecimento de perda auditiva ${ }^{(13,14)}$.

O Déficit do Processamento Auditivo (DPA) pode ser definido como um grupo complexo e heterogêneo de alterações usualmente associado a uma série de dificuldades auditivas e de aprendizado, porém havendo normalidade da audição periférica $^{(15)}$.

Existem vários processos relacionados à audição, tais como atenção (habilidade do indivíduo de preparar, focar um estímulo sonoro, e ainda estar pronto para receber um estímulo diferente em qualquer tempo); deteç̧ão (habilidade de receber estímulos); discriminação (habilidade de resolução de frequência, intensidade, duração); localização (habilidade de analisar diferenças de tempo e de intensidade dos sons recebidos e emitidos por cada um dos lados da orelha); identificação (habilidade de selecionar estímulos e ignorar outros, também denominada de atenção seletiva, e/ou habilidade de partilhar a atenção em dois estímulos, também denominada de atenção dividida); memória (habilidade de memorizar um padrão de frequência ou padrão de duração de sons ou padrão de dois ou mais sons) e compreensão (habilidade de interpretação dos eventos sonoros integrando-os com as outras informações sensoriais) ${ }^{(13)}$.

Algumas áreas contribuíram para a identificação das habilidades auditivas, e dentre elas destaca-se a psicologia, que descreve a habilidade de figura-fundo, como a habilidade de selecionar o estímulo auditivo principal na presença de outros estímulos ruidosos ou de fala; e o fechamento auditivo, identificado como a habilidade de perceber a informação completa quando alguma parte é omitida ${ }^{(16,17)}$.

A habilidade para produzir fala inteligível depende, em grande parte, das habilidades para processar os paradigmas de espectro acústico e da prosódia da fala do locutor. A transformação do sinal sonoro verbal em representações internas dos sons da fala, com padrões organizados e com significado, depende do conhecimento da língua que o indivíduo tem ${ }^{(16-18)}$.

Desta forma, de acordo com as premissas relatadas, este artigo tem por objetivo investigar a relação entre as habilidades auditivas e as combinações de traços presentes na fala de crianças com desvios fonológicos.

\section{MÉTODOS}

A presente pesquisa é do tipo transversal, prospectiva, na qual o desvio fonológico é variável dependente e o resultado dos testes do PA e os traços ou combinação de traços ausentes na fala das crianças constituem as variáveis independentes.

Este estudo foi realizado a partir de dados clínicos de crianças participantes do projeto de pesquisa "Estudo dos desvios fonológicos: classificação e avaliação", aprovado no Comitê de Ética em Pesquisa da Universidade Federal de Santa Maria sob número 0093.0.243.000-09.

Os dados foram coletados no segundo semestre de 2009, especificamente entre os meses de agosto e setembro. As avaliações foram aplicadas em dias distintos, em sessões de quarenta e cinco minutos para a coleta da fala e de uma hora e trinta minutos para avaliação do processamento auditivo (caso o paciente mostrasse indisposição, era marcado retorno para o dia seguinte).

Para os sujeitos fazerem parte da amostra, foram considerados os seguintes critérios de inclusão: estarem autorizados pelos pais e/ou responsáveis para a participação na pesquisa por meio da assinatura do Termo de Consentimento Livre e Esclarecido; as crianças deveriam concordar em participar do estudo, ter diagnóstico de desvio fonológico, apresentar dominância lateral direita (destro) e ter idade superior a 5 anos.

Como critério de exclusão considerou-se os casos de: alterações evidentes nos aspectos neurológico, cognitivo e/ ou psicológico, presença de perda auditiva ou alteração do sistema estomatognático que pudessem estar relacionados com as alterações de fala; fazer uso de instrumentos musicais, ter 
realizado tratamento fonoaudiológico anterior ou apresentar dificuldade de concentração detectada durante a triagem.

Para a seleção da amostra, foram avaliadas crianças selecionadas a partir do setor de triagens de um serviço público de atendimento fonoaudiológico, vinculado a uma Instituição de Ensino Superior. O número de sujeitos da pesquisa baseou-se na demanda anual de atendimento fonoaudiológico apresentada na instituição de origem.

Foi realizada uma nova triagem fonoaudiológica, incluindo as avaliações de linguagem, voz e sistema estomatognático. As crianças também passaram por avaliação audiológica para pesquisa dos limiares e para descartar a presença de perda auditiva. Caso fosse necessário, depois do contato inicial, as crianças foram encaminhadas para avaliações otorrinolaringológica, psicológica e neurológica.

Passaram pela triagem um total de 35 crianças. Contudo, atenderam aos critérios de inclusão do grupo estudo 22 sujeitos, com idades entre 5 e 7 anos e média de idade de 6 anos e 3 meses, para comporem a amostra, sendo que deste total, dez sujeitos eram do gênero masculino enquanto que 12 eram do gênero feminino.

Para a coleta de dados foi realizada a avaliação da fonologia com a aplicação do instrumento Avaliação Fonológica da Criança (AFC) $)^{(1)}$. Este instrumento é constituído de cinco desenhos temáticos (banheiro, cozinha, sala, veículos, e zoológico) que possibilitam a obtenção de uma amostra de fala, por meio de nomeação e fala espontânea.

Os dados de fala foram gravados em sala silenciosa e depois transcritos e revistos por mais dois julgadores fonoaudiólogos, utilizando a análise perceptiva auditiva. Após a transcrição dos dados foi realizada a análise contrastiva.

Para considerar os fonemas adquiridos e alterados tomou-se em consideração um fonema adquirido (produção igual ou superior a $80 \%)$, parcialmente adquirido (40\% a $79 \%$ de produção) e fonema não adquirido (igual ou inferior a $39 \%)^{(19)}$. Depois, por meio do Modelo Implicacional de Complexidade de Traços (MICT), verificou-se os traços ou as combinações de traços distintivos que compõem os fonemas considerados ausentes.

Também foi descrita a porcentagem de alterações dos traços ou combinações de traços de acordo com as possibilidades de cada nível de complexidade.

Foi realizado o cálculo da Porcentagem de Consoantes Corretas Revisado (PCC-R) para identificar o nível de gravidade do desvio fonológico. Foi utilizado o critério do PCC- $\mathbf{R}^{(20)}$ em que não se considera as distorções como erro. Este índice considera o desvio de grau médio (DM) entre 86 e 100\%, de produção correta, o desvio de grau médio-moderado (DMM) entre 66 e $85 \%$, o desvio de grau moderado-severo (DMS) entre 51 e $65 \%$ e o desvio de grau severo (DS) menor que $50 \%$.

Ainda como procedimento de coleta de dados foi realizada a avaliação do procedimento auditivo. Para verificar as habilidades auditivas foi realizada a Avaliação Simplificada do Processamento Auditivo, o Teste de Fusão Binaural, o Teste Dicótico de Dissílabos Alternados, o Teste Dicótico de Dígitos e o Teste de Fala no Ruído presentes no Processamento Auditivo Central: Manual de aplicação ${ }^{(14)}$. Para a aplicação de cada prova, levou-se em consideração a idade padrão em que as habilidades auditivas se desenvolvem, bem como a indicação da literatura para a aplicação de determinado teste por idade.

A Avaliação Simplificada do Processamento Auditivo (Triagem) consiste em três tarefas, sendo a primeira de três sequências de sons não-verbais, a segunda de três sequências de sons verbais e por último a tarefa de localização sonora em cinco direções (são avaliadas as habilidades auditivas de Localização e Ordenação temporal simples).

O Teste de Fusão Binaural (FB) consiste em uma lista de palavras monossílabas distorcidas acusticamente (habilidade auditiva de Síntese Binaural).

O Teste Dicótico de Dissílabos Alternados (SSW) envolve uma lista de palavras faladas de forma livre e de forma competitiva: Direita livre $\rightarrow$ direita competitiva $\rightarrow$ esquerda competitiva $\rightarrow$ esquerda livre (habilidades auditivas de ordenação temporal complexa e figura-fundo para sons verbais).

No Teste Dicótico de Dígitos (DD) é utilizada uma lista de palavras (representando números) apresentada simultaneamente de forma dicótica. O paciente deve prestar atenção, sendo solicitado a dizer tudo o que escutou. Depois o paciente deve responder somente o que escutou de um lado, ignorando o que é dito na outra orelha (habilidade auditiva de figura-fundo para sons verbais em processo de atenção sustentada).

O Teste de Fala no Ruído (FR) é composto por uma lista de palavras apresentadas simultaneamente com o ruído branco, de forma ipsi ou contralateral (Fechamento Auditivo);

O PSI em Português é um teste composto por dez frases que mostram ações e a criança deve apontar a figura correspondente à ação que escutou. Cada frase é apresentada ao mesmo tempo em que, de forma competitiva ipsilateralmente, uma estória é contada.

Todos os testes foram realizados em cabine acusticamente tratada, sem interferência do meio, e foram aplicados com Audiômetro Clínico Fonix FA - 12, e uso de fone TDH 39 (calibração ANSI S3.6/96: ANSI S343/92; ISSO 389/91).

A análise estatística utilizada foi realizada por meio do programa STATA versão 10.1 e considerou-se significativos valores abaixo de 5\%, nas correlações do PA com as combinações de traços. Também foi analisado o PA em relação à gravidade do desvio fonológico, e às variáveis extra-linguísticas gênero e idade.

\section{RESULTADOS}

Todos os sujeitos tiveram êxito de $100 \%$ no teste PSI em Português, portanto, seus resultados não entraram na análise aqui descrita.

Assim, na Tabela 1 está apresentado o desempenho de acertos nos testes do PA, por meio da média e do desvio-padrão.

A Tabela 2 demonstra o número de alterações possíveis em cada combinação de traços distintivo do MICT. A porcentagem de alterações foi obtida por meio da divisão do número de alterações de cada traço (de acordo com o número total de sujeitos) pelo número total de possibilidades (considerando todos os sujeitos) multiplicado por 100, a fim de obter a porcentagem correspondente.

É possível notar que a combinação de traços [+aprox, +cont] que corresponde ao fonema $/ \mathrm{r} /$ é o que possui maior porcentagem de alterações. 
Tabela 1. Desempenho de acertos nos testes do processamento auditivo

\begin{tabular}{lccc}
\hline $\begin{array}{l}\text { Teste do processamento } \\
\text { auditivo }\end{array}$ & $\begin{array}{c}\text { Tarefa dos } \\
\text { testes }\end{array}$ & Médias & DP \\
\hline \multirow{2}{*}{ Triagem } & SNV & 2,10 & 0,73 \\
& SV & 2,90 & 1,19 \\
& LS & 4,04 & 0,95 \\
\hline \multirow{2}{*}{ Dicótico de dígitos } & AL & $3, .22$ & 8,70 \\
& AD & $2, .27$ & 10,12 \\
Fala no ruído & AE & 19,77 & 8,10 \\
\hline \multirow{2}{*}{ Fusão binaural } & OD & 90,36 & 7,37 \\
& OE & 87,81 & 9,19 \\
\hline \multirow{2}{*}{ Dissílabos alternados (SSW) } & OD & 94,72 & 5,57 \\
& OE & 91,81 & 7,35 \\
\hline
\end{tabular}

Legenda: $\mathrm{SNV}$ = sequência não-verbal; $\mathrm{SN}$ = sequência verbal; $\mathrm{LS}$ = localização sonora; $\mathrm{AL}=$ atenção livre; $\mathrm{AD}$ = atenção à direita; $\mathrm{AE}=$ atenção à esquerda; $\mathrm{OD}=$ orelha direita; $\mathrm{OE}=$ orelha esquerda; $\mathrm{DNC}=$ direita não-competitiva; $\mathrm{DC}=$ direita competitiva; $\mathrm{EC}$ = esquerda competitiva; $\mathrm{ENC}$ = esquerda não competitiva; $\mathrm{DP}=$ desvio-padrão
Quanto ao resultado de acertos das tarefas do PA correlacionando com as combinações de traços alterados, tem-se a Tabela 3.

Nesta tabela foram colocadas apenas as correlações em que o nível de significância foi menor que 5\%, mesmo não havendo uma correlação forte entre as variáveis.

Já a Tabela 4 apresenta os resultados de acertos nos testes de processamento em relação ao grau de gravidade do desvio fonológico.

Nesta tabela estão representados os valores obtidos pelos sujeitos com diferentes níveis de gravidade do desvio fonológico, de acordo com o teste de PA. Foi também descrito o valor da significância, o qual, somente o valor foi menor que 0,05 no subteste AE-OE do Dicótico de Dígitos, e a resposta foi dada ipsilateralmente ao solicitado.

Por fim, na Tabela 5 estão apresentados os valores de acertos dos testes do PA e as variáveis extralinguísticas gênero e idade.

Essa tabela indica os valores de acertos obtidos pelos sujeitos de ambos os gêneros e nas três faixas etárias avaliadas, de acordo com o teste do PA. Nota-se também que apenas na variável idade houve significância menor que 5\% em relação a algum dos testes, sendo este o subteste AE-OD do Dicótico de Dígitos, em que a resposta é dada de forma contralateral ao solicitado.

Tabela 2. Distribuição dos traços de acordo com as alterações apresentadas e os fonemas correspondentes

\begin{tabular}{|c|c|c|}
\hline Combinações de traços por nível segundo o MICT & $\begin{array}{c}\text { Fonemas correspondentes em } \\
\text { cada nível }\end{array}$ & $\begin{array}{c}\text { Frequência/possibilidade de ocorrência } \\
\text { (Percentual de alterações - \%) }\end{array}$ \\
\hline Estado 0 & $/ \mathrm{m}, \mathrm{n}, \mathrm{p}, \mathrm{t} /$ & $6 / 88(6,81)$ \\
\hline Nível 1: [-anterior] & $/ \mathrm{h} /$ & $1 / 22(4,54)$ \\
\hline Nível 2: [+vozeado] & $/ b, d /$ & $15 / 44(34,07)$ \\
\hline Nível 3: [dorsal]/[-vozeado] & $/ \mathrm{k} /$ & $2 / 22(9,09)$ \\
\hline Nível 4: [dorsal,+vozeado] & $\lg /$ & $10 / 22(45,45)$ \\
\hline Nível 5: [+contínuo]/[ \pm vozeado] & $/ f, v, s, z /$ & $31 / 44(35,22)$ \\
\hline Nível 6: [+aproximante] & $/ / /$ & $6 / 22(27,22)$ \\
\hline Nível 7: [coronal,+contínuo]/(-anterior) & $/ \int, 3 /$ & $30 / 44(68,18)$ \\
\hline Nível 8: [+aproximante,+contínuo] & $/ \mathrm{r} /$ & $19 / 22(86,36)$ \\
\hline Nível 9a: [+aproximante,+contínuo,dorsal] & $/ \mathrm{R} /$ & $4 / 22(18,18)$ \\
\hline Nível 9b: [+aproximante,-anterior] & $/ N$ & $13 / 22(59,09)$ \\
\hline
\end{tabular}

Tabela 3. Relação do processamento auditivo com a combinação de traços distintivos, baseado nos erros de fala e acertos no processamento auditivo

\begin{tabular}{lcccccc}
\hline Testes do processamento auditivo & \multicolumn{5}{c}{ Traços ou combinação de traços distintivos } \\
\cline { 3 - 6 } & & {$[-$ ant $]$} & {$[+$ cont] $( \pm$ voz $)$} & {$[+$ aprox $]$} & {$[+$ +aprox,+cont] } & {$[+$ aprox,-ant] } \\
\hline Triagem & SNV & - & - & - & 0,46 & - \\
Dicótico de dígitos & AL-OD & - & - & - & 0,43 & - \\
Fusão binaural & OD & $-0,43$ & - & - & - & - \\
& OE & - & - & $-0,44$ & - \\
SSW & DNC & - & $-0,42$ & - & - \\
\hline
\end{tabular}

Legenda: SNV = sequência não-verbal; $\mathrm{AL}$ = atenção livre; OD = orelha direita; OE = orelha esquerda; $\mathrm{DNC}=$ direita não-competitiva; [-ant] = menos anterior; $[+\mathrm{cont}]( \pm \mathrm{VOz})=$ mais contínuo e mais ou menos vozeado; $[$ +aprox $]$ = mais aproximante; $[$ +aprox,+cont $]$ = mais aproximante e mais contínuo; $[+$ aprox,-ant $]$ = mais aproximante e menos anterior 
Tabela 4. Relação dos testes do processamento auditivo com o grau de gravidade do desvio fonológico

\begin{tabular}{|c|c|c|c|c|c|c|}
\hline \multirow{2}{*}{\multicolumn{2}{|c|}{ Testes do processamento auditivo }} & \multicolumn{4}{|c|}{ Grau do desvio fonológico } & \multirow[b]{2}{*}{ Valor de $p$} \\
\hline & & \multirow{2}{*}{$\begin{array}{c}\text { Médio } \\
(n=8)\end{array}$} & \multirow{2}{*}{$\begin{array}{c}\text { Médio-moderado } \\
(\mathrm{n}=9)\end{array}$} & \multirow{2}{*}{$\begin{array}{c}\text { Moderado-severo } \\
(\mathrm{n}=2)\end{array}$} & $\begin{array}{c}\text { Severo } \\
(n=3)\end{array}$ & \\
\hline & SNV & & & & 1,00 & 0,24 \\
\hline \multirow[t]{2}{*}{ Triagem } & SV & 2,56 & 1,84 & 1,32 & 2,00 & 0,19 \\
\hline & TLS & 4,00 & 4,44 & 3,33 & 3,66 & 0,30 \\
\hline \multirow{6}{*}{ Dicótico de dígitos } & AL-OD & 21,00 & 20,44 & 23,66 & 13,33 & 0,12 \\
\hline & AL-OE & 19,00 & 21,67 & 25,66 & 14,66 & 0,18 \\
\hline & AD-OD & 15,71 & 21,00 & 28,33 & 16,67 & 0,31 \\
\hline & AD-OE & 14,42 & 17,44 & 5,33 & 6,00 & 0,59 \\
\hline & AE-OE & 20,00 & 20,33 & 30,67 & 10,00 & $0,009^{*}$ \\
\hline & AE-OD & 12,00 & 13,67 & 9,33 & 12,00 & 0,83 \\
\hline \multirow{2}{*}{ Fusão binaural } & OD & 93,71 & 90,22 & 89,33 & 84,00 & 0,30 \\
\hline & OE & 92,57 & 88,44 & 82,66 & 80,00 & 0,17 \\
\hline \multirow{2}{*}{ Fala no ruído } & OD & 97,85 & 96,00 & 94,67 & 90,67 & 0,59 \\
\hline & OE & 94,28 & 92,44 & 90,67 & 85,33 & 0,37 \\
\hline \multirow{4}{*}{ SSW } & DNC & 16,28 & 19,55 & 19,33 & 22,67 & 0,37 \\
\hline & DC & 20,85 & 25,55 & 28,66 & 27,33 & 0,12 \\
\hline & EC & 21,71 & 22,11 & 26,00 & 22,67 & 0,55 \\
\hline & ENC & 17,00 & 16,78 & 20,00 & 18,67 & 0,81 \\
\hline
\end{tabular}

*Valores significativos $(\mathrm{p} \leq 0,05)$ - Teste ANOVA

Legenda: $S N V$ = sequência não-verbal; $S N=$ sequência verbal; $L S$ = localização sonora; $A L=$ atenção livre; $A D=$ atenção à direita; $A E$ = atenção à esquerda; $O D$ = orelha direita; $\mathrm{OE}$ = orelha esquerda; $\mathrm{DNC}=$ direita não-competitiva; $\mathrm{DC}$ = direita competitiva; $\mathrm{EC}=$ esquerda competitiva; $\mathrm{ENC}=$ esquerda não competitiva; $\mathrm{n}$ = número de sujeitos

Tabela 5. Relação dos testes do processamento auditivo com as variáveis gênero e idade

\begin{tabular}{|c|c|c|c|c|c|c|c|c|}
\hline \multirow{2}{*}{\multicolumn{2}{|c|}{ Testes do processamento auditivo }} & \multicolumn{2}{|c|}{ Gênero } & \multirow{2}{*}{ Valor de $p$} & \multicolumn{3}{|c|}{ Idade } & \multirow{2}{*}{ Valor de $\mathrm{p}$} \\
\hline & & Masculino & Feminino & & 5 anos & 6 anos & 7 anos & \\
\hline \multirow{3}{*}{ Triagem } & SNV & 2,00 & 1,60 & 0,37 & 1,45 & 2,00 & 2,33 & 0,21 \\
\hline & SV & 2,53 & 2,04 & 0,65 & 2,85 & 2,20 & 2,70 & 0.69 \\
\hline & TLS & 4,08 & 4,00 & 0,84 & 3,82 & 4,40 & 4,17 & 0,51 \\
\hline \multirow{6}{*}{ Dicótico de dígitos } & AL-OD & 21,00 & 19,00 & 0,43 & 17,63 & 21,00 & 23,83 & 0,88 \\
\hline & AL-OE & 21,83 & 18,70 & 0,27 & 20,64 & 15,60 & 24,00 & 0,10 \\
\hline & $A D-O D$ & 21,58 & 17,50 & 0,36 & 16,36 & 21,20 & 24,67 & 0,26 \\
\hline & $A D-O E$ & 13,08 & 13,50 & 0,91 & 14,63 & 16,20 & 8,33 & 0,25 \\
\hline & $A E-O E$ & 22,00 & 18.10 & 0,27 & 18,91 & 16,60 & 25,67 & 0,13 \\
\hline & $A E-O D$ & 11,08 & 13,80 & 0,36 & 11,45 & 19,20 & 8,16 & $0,031^{*}$ \\
\hline \multirow{2}{*}{ Fusão binaural } & OD & 91,33 & 89,20 & 0,51 & 93,81 & 87,2 & 86,66 & 0,82 \\
\hline & OE & 89,00 & 86,40 & 0,52 & 91,27 & 84,00 & 84,67 & 0,22 \\
\hline \multirow{2}{*}{ Fala no ruído } & OD & 93,33 & 96,40 & 0,21 & 94,18 & 96,80 & 94,00 & 0,66 \\
\hline & $\mathrm{OE}$ & 92,33 & 91,20 & 0,73 & 90,18 & 93,60 & 93,33 & 0,60 \\
\hline \multirow{4}{*}{ SSW } & DNC & 19,50 & 18,20 & 0,59 & 18,91 & 21,00 & 17,20 & 0,53 \\
\hline & DC & 23,67 & 26,00 & 0,35 & 26,36 & 24,20 & 22,17 & 0,35 \\
\hline & $\mathrm{EC}$ & 21,91 & 23,40 & 0,43 & 24,54 & 21,60 & 19,83 & 0,80 \\
\hline & DEC & 18,83 & 16,00 & 0,21 & 19,27 & 14,60 & 16,83 & 0,24 \\
\hline
\end{tabular}

*Valores significativos $(\mathrm{p} \leq 0,05)-$ Teste ANOVA

Legenda: SNV = sequência não-verbal; $S N=$ sequência verbal; $L S$ = localização sonora; $A L$ = atenção livre; $A D$ = atenção à direita; $A E$ = atenção à esquerda; $\mathrm{OD}=$ orelha direita; $\mathrm{OE}=$ orelha esquerda; $\mathrm{DNC}=$ direita não-competitiva; $\mathrm{DC}=$ direita competitiva; $\mathrm{EC}=$ esquerda competitiva; $\mathrm{ENC}=$ esquerda não competitiva

\section{DISCUSSÃO}

A aquisição de fala em crianças com desvio é estudada há muito tempo, e sua etiologia é bastante explorada ${ }^{(9)}$. Entretanto, é consenso que a alteração do PA pode favorecer o seu desenvolvimento, visto que a via auditiva é essencial na aquisição da linguagem ${ }^{(12,17,20-22)}$.

Estudos que relacionem as alterações do PA com a aquisição de fala ainda são muito escassos, principalmente no que diz respeito à aquisição dos traços distintivos ${ }^{(20,22-24)}$.

Por meio do MICT, foi possível notar que a maior parte das alterações são encontradas a partir do nível 2 ([+voz]), o que coincide com outro estudo ${ }^{(25)}$. Entretanto, foi possível encontrar três alterações nas combinações de traços no estado 0 e uma alteração no nível 1 ([-ant]).

A correlação do PA com as combinações de traços mostrou valores entre $40 \%$ e $50 \%$, apesar de os valores serem significantes $(\mathrm{p} \leq 0,05)$. Estas correlações, apesar de fracas, demonstram 
uma relação do PA com os traços.

A sequência de sons verbais, da triagem, foi o que mostrou correlação mais alta com alguma das combinações de traços, no caso a [+aprox, +cont], que corresponde ao fonema $/ \mathrm{r} /$. Esse subteste permite avaliar a capacidade de ordenação temporal. Mesmo não sendo uma correlação forte $(0,46 \%)$, este achado mostra uma tendência de que crianças com alteração nesta combinação de traços pode também apresentar déficit nesta habilidade auditiva.

Crianças que apresentam o sistema auditivo central ainda imaturo, como evidenciado nos testes SSW e dicótico de dígitos, podem ter ausência do fonema /r/, já que, de acordo com a literatura ${ }^{(1,8,26,27)}$, esse é o fonema que apresenta estabelecimento mais tardio, e também o maior número de alterações em crianças com desvios fonológicos.

A habilidade de ordenação temporal, evidenciada no teste SSW e na triagem, pela subtarefa SNV, mostra ser a habilidade que mais pode estar comprometida em crianças com alteração de traços distintivos.

Observando os traços de forma isolada, nota-se que as porcentagens de alterações estão mais elevadas em combinações de traços que possuem o traço [+contínuo], que participa da composição de vários fonemas do Português Brasileiro, principalmente os fonemas fricativos. Este traço se refere ao modo de articulação do som, e ocorre quando há uma constrição do trato vocal não tão estreitada a ponto de bloquear o fluxo aéreo.

Em se tratando das fricativas, é possível perceber um maior envolvimento dos percentuais de alteração apresentando valores altos (depois do fonema /r/). Desta forma, acredita-se que estes sejam os fonemas mais acometidos pelo DPA.

Assim, a combinação de traços [coronal,+contínuo]/(-anterior), que compõe os fonemas $/ \int, 3 /$, apresentou um valor elevado de alterações $(68,18 \%)$. Esta combinação pode sofrer influencia do DPA.

Quanto ao grupo das liquidas, o fonema $/ K /$ também apresentou muitas alterações em sua combinação de traços [+aproximante,-anterior]. Este também é um fonema tardio na aquisição fonológica, bem como o /r/.

É possível notar, portanto, analisando novamente os traços de forma isolada, que os traços [+aproximante] e o traço [-anterior] também têm tendência a apresentar alterações em crianças com DPA, assim como o traço [+contínuo].

Ao analisar o PA com as variáveis extralinguísticas inerentes aos sujeitos, como o gênero e a idade nota-se não haver uma correlação significativa, já que o valor de significância não está abaixo de 5\%, com exceção da subtarefa AE-OD, na qual a resposta é contralateral ao solicitado ao paciente. Pode-se dizer que há alguma dificuldade de manter a atenção à orelha direita nesta população.

Não foram encontrados na literatura pesquisada trabalhos que permitam discutir os dois achados relatados anteriormente, portanto, novos estudos irão favorecer o reconhecimento destes.

Em se tratando das habilidades auditivas, de acordo com os achados aqui descritos, temos que o teste SSW e o Dicótico de Dígitos são os que apresentaram piores resultados, o que indica que as habilidades de ordenação temporal complexa e de figura-fundo são as que mais podem estar comprometidas na população aqui estudada.

O teste SSW mostrou pior resultado nas subtarefas EC e DC, sendo esta tarefa que descreve melhor a habilidade de figura-fundo, evidenciada pelo teste Dicótico de Dígitos. A presença de pior desempenho nessa subtarefa é corroborada por vários estudos recentes ${ }^{(28,29)}$, tanto em crianças com aquisição de fala normal quanto em crianças com desvios.

Ainda em se tratando do teste SSW, é importante salientar os tipos de erros que mais ocorreram. As inversões e as omissões foram as mais frequentes, em proporção, para os acertos, de 5/1 e 4/1 respectivamente, o que pode caracterizar um déficit nos processos de decodificação e codificação. Este achado também é relatado em outro estudo, entretanto, com população com idade mais elevada ${ }^{(28)}$.

O processo de decodificação associa-se à aquisição de informações a respeito dos fonemas da língua, estando, portanto, diretamente relacionado com a população deste estudo. Já o processo de codificação pode ser associado à aquisição de informações a respeito das regras de significação da língua, memórias sintática, semântica e fonológica. Sendo assim, caso não haja a aquisição de acordo com o padrão adulto, pode ocorrer o estabelecimento dos desvios fonológicos. Estes achados permitem dizer que há um déficit de memória sensorial do código da língua ${ }^{(28,30)}$.

Quanto ao teste Dicótico de Dígitos, há uma leve diferença entre as subtarefas, com pior desempenho na atenção livre. Quando as crianças foram solicitada a manter a atenção à orelha esquerda, houve uma leve melhora. Esta diferença evidencia o déficit da habilidade auditiva em questão, a figurafundo, esta que permite ao indivíduo manter a atenção sobre um estímulo em detrimento de outro, podendo ocorrer tanto de forma ipsi quanto contralateral.

Existe uma relação causal entre problemas de linguagem e DPA, sobretudo no aspecto da compreensão da linguagem oral. O desenvolvimento da fala é influenciado pelas capacidades perceptivas da criança. É por meio da recepção, da análise e da organização do processamento das informações auditivas que se estabelece a representação mental do estímulo linguístico e o armazenamento dessa representação na memória ${ }^{(30)}$.

Este estudo permite vislumbrar a possível relação das alterações do PA com as combinações de traços em crianças com aquisição de fala desviante. No entanto, novos estudos e um aprofundamento maior seriam interessantes e necessários.

\section{CONCLUSÃO}

Este artigo mostrou que as combinações de traços em crianças com aquisição de fala desviante, apresentam-se alteradas, e que o DPA pode influenciar esta alteração, principalmente aquelas em que há o traço [+contínuo].

Os resultados obtidos nos testes SSW e Dicótico de Dígitos mostraram haver alteração nas habilidades de ordenação temporal complexa e figura-fundo, bem como memória sensorial defasada. 


\begin{abstract}
Purpose: To investigate the relationship between auditory abilities and combinations of distinctive features present in the speech of children with phonological disorders. Methods: Participants were 22 children diagnosed with phonological disorders, of both genders, with ages between 5 and 7 years. Speech data were gathered using the Phonological Assessment of Children. The combination of features was observed through the Implicational Model Complexity of Traces (MICT). The acquired features considered a correct production above $80 \%$. The following tests were also applied: Simplified Auditory Processing Evaluation (screening), Staggered Spondaix Word Test (SSW), Dichotic Listening Test, Binaural Fusion Test, Speech-in-Noise Test, and Pediatric Speech Intelligibility (PSI). Results: All children had 100\% success in the PSI. In the Binaural Fusion and Speech-in-Noise tests, as well as in the screening, subjects also had high averages. The SSW and the Dichotic Listening Test showed low scores. The combination of features that was more affected was [+approximant,+continuous], which is in the composition of the phoneme /r/, followed by [coronal,+continuous]/(-front), which compose the phonemes / $/ 3 /$. Conclusion: The results obtained in the SSW and Dichotic Listening Test showed a deficit in the abilities of complex temporal order and figure-ground, as well as lagged sensorial memory. These deficits might jeopardize the acquisition of the features described.
\end{abstract}

Keywords: Speech disorders; Hearing disorders; Auditory perception; Auditory perceptual disorders; Hearing tests; Child

\section{REFERÊNCIAS}

1. Yavas MS, Hernandorena CL, Lamprecht RR. Avaliação fonológica da criança: reeducação e terapia. Porto Alegre: Artes Médicas; 1992.

2. Vieira MG, Mota HB, Keske-Soares M. Relação entre idade, grau de severidade do desvio fonológico e consciência fonológica. Rev Soc Bras Fonoaudiol. 2004;9(3):144-50.

3. Spíndola RA, Payão LM, Bandini HH. Abordagem fonoaudiológica em desvios fonológicos fundamentada na hierarquia de traços distintivos e na consciência fonológica. Rev CEFAC. 2007;9(2):180-9.

4. Mota HB. Aquisição segmental do português: um modelo implicacional de complexidade de traços [tese]. Porto Alegre: Faculdade de Letras da Pontifícia Universidade Católica do Rio Grande do Sul; 1996.

5. Papp AC, Wertzner HF. O aspecto familial e o transtorno fonológico. Pró-Fono. 2006;18(2):151-60.

6. Pagan LO, Wertzner HF. Análise acústica das consoantes líquidas do Português Brasileiro em crianças com e sem transtorno fonológico. Rev Soc Bras Fonoaudiol. 2007;12(2):106-13.

7. Pereira LD. Sistema auditivo e desenvolvimento das habilidades auditivas. In: Ferreira LP, Befi-Lopes DM, Limongi SCO. Tratado de fonoaudiologia. São Paulo: Roca; 2004. p.547-52.

8. Wertzner HF, Oliveira MM. Semelhanças entre os sujeitos com distúrbio fonológico. Pró-Fono. 2002;14(2):143-52.

9. Lewis BA, Freebairn LA, Hansen AJ, Stein CM, Shriberg LD, Iyengar SK, Gerry Taylor H. Dimensions of early speech sound disorders: a factor analytic study. J Commun Disord. 2006;39(2):139-57.

10. Rvachew S, Grawburg M. Correlates of phonological awareness in preschoolers whit speech sound disorders. J Speech Lang Hear Res. 2006;49(1):74-87.

11. Musiek FE. Aplicação de testes auditivos centrais: uma abordagem geral. In: Katz J, editor. Tratado de audiologia clínica. São Paulo: Manole; 1989. p. 323-39.

12. Carroll JM, Snowling MJ. Language and phonological skills in children at high risk of reading difficulties. J Child Psychol Psychiatry. 2004:45(3):631-40.

13. Jerger J, Musiek F. Report of the Consensus Conference on the Diagnosis of Auditory Processing Disorders in School-Aged Children. J Am Acad Audiol. 2000;11(9):467-74.

14. Pereira LD, Schochat E. Processamento auditivo central: manual de avaliação. São Paulo: Lovise; 1997.

15. Zalcman TE, Schochat E. A eficácia do treinamento auditivo formal em indivíduos com transtorno de processamento auditivo. Rev Soc Bras Fonoaudiol. 2007;12(4):310-4.
16. Heath SM, Hogben JH, Clark CD. Auditory temporal processing in disabled readers with and without oral language delay. J Child Psychol Psychiatry. 1999;40(4):637-47.

17. Murphy CF, Schochat E. How auditory temporal processing deficits relate to dyslexia. Braz J Med Biol Res. 2009;42(7):647-54.

18. Bernhardt B. The application of nonlinear phonological theory to intervention with one phonologically disordered child. Clin Linguist Phon. 1992;6(4):283-316.

19. Shriberg LD, Austin D, Lewis BA, McSweeny JL, Wilson DL. The speech disorders classification system (SDCS): extensions and lifespan reference data. J Speech Lang Hear Res. 1997;40(4):723-40.

20. Bamiou DE, Musiek FE, Luxon LM. Aetiology and clinical presentations of auditory processing disorders- a review. Arch Dis Child. 2001;85(5):361-5.

21. Chermak GD, Silva ME, Nye J, Hasbrouck J, Musiek FE. An update on professional education and clinical practices in central auditory processing. J Am Acad Audiol. 2007;18(5):428-52; quiz 455.

22. Cooper DH, Roth FP, Speece DL, Schatschneider C. The contribution of oral language skills to the development of phonological awareness. Appl Psycholing. 2002;23(3):399-416.

23. Tallal P. Auditory temporal perception, phonics, and reading disabilities in children. Brain Lang. 1980;9(2):182-98.

24. Stoel-Gammon C. Normal and disordered phonology in two-year-olds. Top Lang Disord. 1991;11(4):21-32.

25. Pereira LF. Desvio fonológico: desempenho de pré-escolares em tarefas linguísticas e metalinguísticas nos diferentes graus de gravidade [tese]. São Paulo: Universidade Federal de São Paulo. Escola Paulista de Medicina; 2006.

26. Lamprecht, RR. Antes de mais nada. In: Lamprecht $\mathrm{R}$, organizadora. Aquisição fonológica do português: perfil de desenvolvimento e subsídios para terapia. Porto Alegre: Artmed; 2004. Porto Alegre: Artmed, 2004.

27. Mezzomo CL, Ribas L. Sobre a aquisição das líquidas. In: Lamprecht $\mathrm{R}$, organizadora. Aquisição fonológica do português: perfil de desenvolvimento e subsídios para terapia. Porto Alegre: Artmed; 2004.

28. Araujo NS, Ruiz AC, Pereira LD. SSW - análise qualitativa dos erros: inventário de atendimento de 2005. Rev CEFAC. 2009;11(Supl 1):44-51.

29. Ferreira MI, Caumo DT. Relação entre desvios fonológicos e processamento auditivo. Rev Soc Bras Fonoaudiol. 2009;14(2):234-40

30. Vitto MM, Feres MC. Distúrbios da comunicação oral em crianças. Medicina (Ribeirão Preto). 2005;38(3/4):229-34. 